\title{
TRANSVERSE SPIN DEPENDENCE OF PP TOTAL CROSS-SECTIONS
}

E. Biegert, J. Buchanan, J. Clement, W. Dragoset, R. Felder, J. Hoftlezer, J. Hudomalj-Gabitzsch, K. Hogstrom, W. Madigan, G. Mutchler, G. C. Phillips, J. Roberts, T. Williams Rice University, Houston, Texas 77001

K. Abe, R. Fernow, T. Mulera University of Michigan, Ann Arbor, Michigan 48109

S. Bart, B. Mayes, L. Pinsky University of Houston, Houston, Texas 77004

This presentation is a brief report of some preliminary results from Experiment 395, which is currently in progress at the ZGS. The main goal of the experiment is to measure the difference between pp total cross-sections in pure initial spin states transverse to the beam direction for incident momenta of 1 to $3 \mathrm{GeV} / \mathrm{c}$ in the laboratory.

The experimental layout is shown in Fig. 3. The incident proton beam is focussed by quadrupoles $Q 1$ and $Q 2$ onto the polarized proton target, PPT V. The PPT has been described by Abe and Mulera earlier at this conference. The beam is defined by counters B1, B2 and B3 and the beam halo is vetoed by counters A1 and A2. Unscattered particles and those scattered at small angles are counted by transmission counters $\mathrm{T} 1-\mathrm{T} 8$, a series of six concentric rings and two disks. Counter $T 9$ is used in the event strobe and to monitor the efficiencles of $\mathrm{T} 1-\mathrm{T} 8$.

Five multiwire proportional chambers (MWPC), P1-P5, are used to reconstruct the incident and scattered rays. Using the known fleld integral of the PPT magnet, the coordinates of the intersection of the two rays are calculated on-1ine in the target plane. The results of this calculation are used to insure that the beam particles pass through the volume of the polarized material. The MWPC data are also used to monitor the beam profile and position and to provide a means of fine-tuning the beam.

The downstream counters are mounted on a movable arm, which pivots about a point under the target. This allows easy repositioning of the counters for each different incident momentum.

The counter signals are processed by fast electronics logic and interfaced to a PDP-11/45 computer via CAMAC. All of the counter scalers and about 10 percent of the MWPC data are displayed and analyzed on-line. All events are written to magnetic tape.

The cross-section difference, $\Delta \sigma_{1}$, for the ith transmission counter is given by

$$
\Delta \sigma_{i}=\left(N_{0} P_{H} L P_{B} P_{T}\right)^{-1} \ln \left[\left(T_{1}(\uparrow \uparrow) / B\right) /\left(T_{i}(\uparrow \downarrow) / B\right)\right]
$$

where $\mathrm{N}_{O}$ is Avogadro's number, $\mathrm{P}_{H}$ and $\mathrm{L}$ are the target density and length, ${ }^{\circ} \mathrm{P}_{\mathrm{B}}$ and $\mathrm{P}_{\mathrm{T}}$ are the beam and target polarizations, $\mathrm{T}_{\mathcal{1}}$ are the counter rates and $B$ is the beam rate. The total cross-section differ- 
ence, $\Delta \sigma_{T}=\Delta \sigma_{T}(\uparrow \downarrow)-\Delta \sigma_{T}(\uparrow \uparrow)$, is obtained by extrapolating the transmission counter data to zero degrees.

The beam spin is reversed on alternate pulses to eliminate longterm instabilities due to beam drift. Other spurious asymmetries are checked by perlodically reversing the target spin. As a final check, data are accumulated using an unpolarized sample, which approximates the target material in composition.

The two preliminary data points obtained to date are plotted in Fig. 1. The data are

$$
\begin{aligned}
& \Delta \sigma_{\mathrm{T}}=5.8 \pm 0.5 \mathrm{mb} \text { at } 2 \mathrm{GeV} / \mathrm{c} \\
& \Delta \sigma \mathrm{T}=3.5 \pm 1.0 \mathrm{mb} \text { at } 1.5 \mathrm{GeV} / \mathrm{c}
\end{aligned}
$$

The point at $2 \mathrm{GeV} / \mathrm{c}$ agrees very well with the previous result of the Michigan group. The lines and solid circles on the graph are predictions of $\Delta \sigma_{T}$ based on the PP phase shifts of MacGregor and Hoshizaki. ${ }^{1,2,3}$ The data are also shown on Fig. 2, a prediction of $\Delta \sigma_{T}$, assuming the difference arises from the inelastic channel pp $\rightarrow$ pn $\pi^{+} .4$

\section{REFERENCES}

1 N. Hoshizaki, et al., Prog. Theor. Phys. 45, 1123 (1971).

2 R. A. Arndt, M. H. MacGregor and R. M. Wright, Phys. Rev. 182, 1714 (1969).

3 G. L. Kane and G. H. Thomas, ANL-HEP-PR-75-56, October 1975.

4 E. L. Berger, ANL-HEP-PR-76-35, May, 1976.

\section{Appendix}

Tabular data for $W$. de Boer et al., Phys. Rev Lett. 34, 558(1975).

\begin{tabular}{cc}
$\mathrm{P}(\mathrm{GeV} / \mathrm{c})$ & \multicolumn{1}{c}{$\Delta \sigma_{\mathrm{T}}(\mathrm{mb})$} \\
2.0 & $5.79 \pm .93$ \\
3.0 & $.76 \pm .20$ \\
4.0 & $.72 \pm .36$ \\
6.0 & $.34 \pm .07$
\end{tabular}




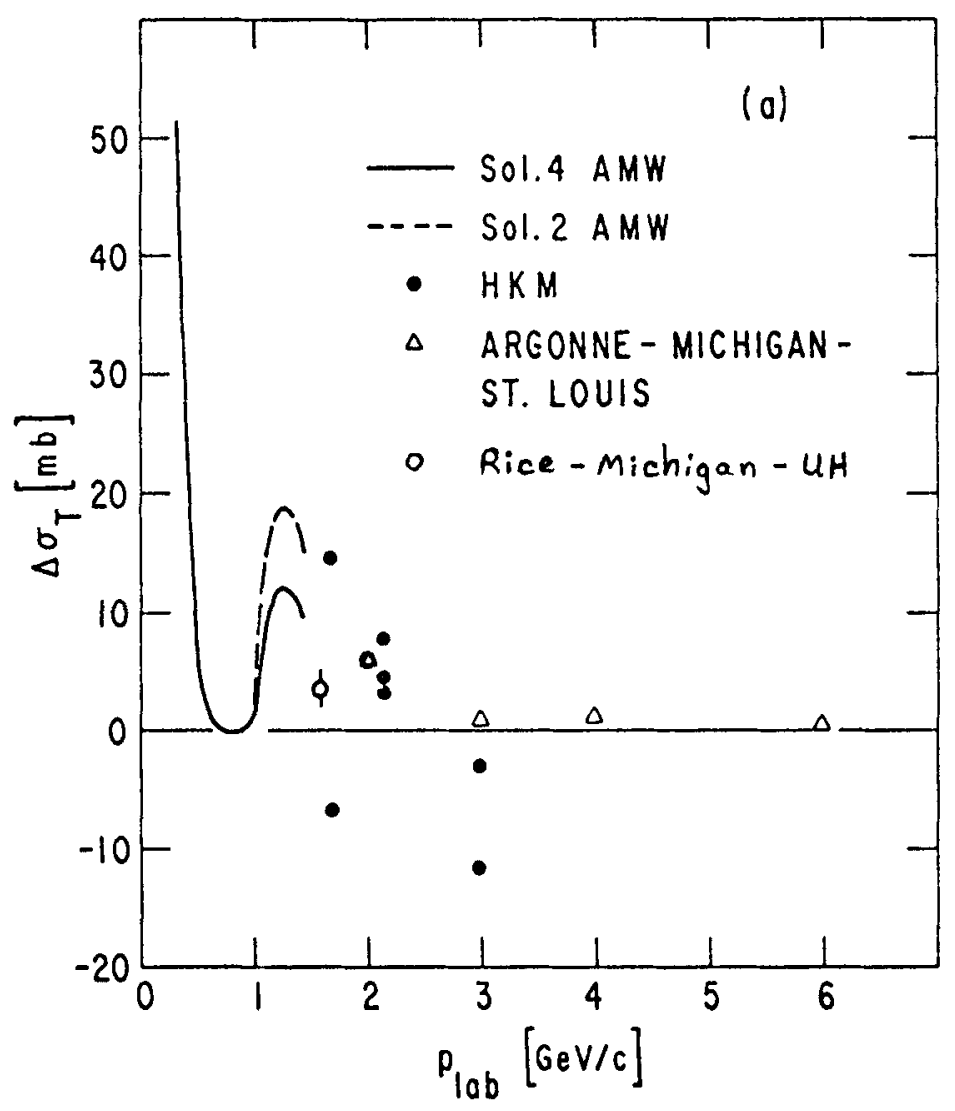

Fig. 1--The new data (open circles) and previous data with predictions calculated from phase shift models. 


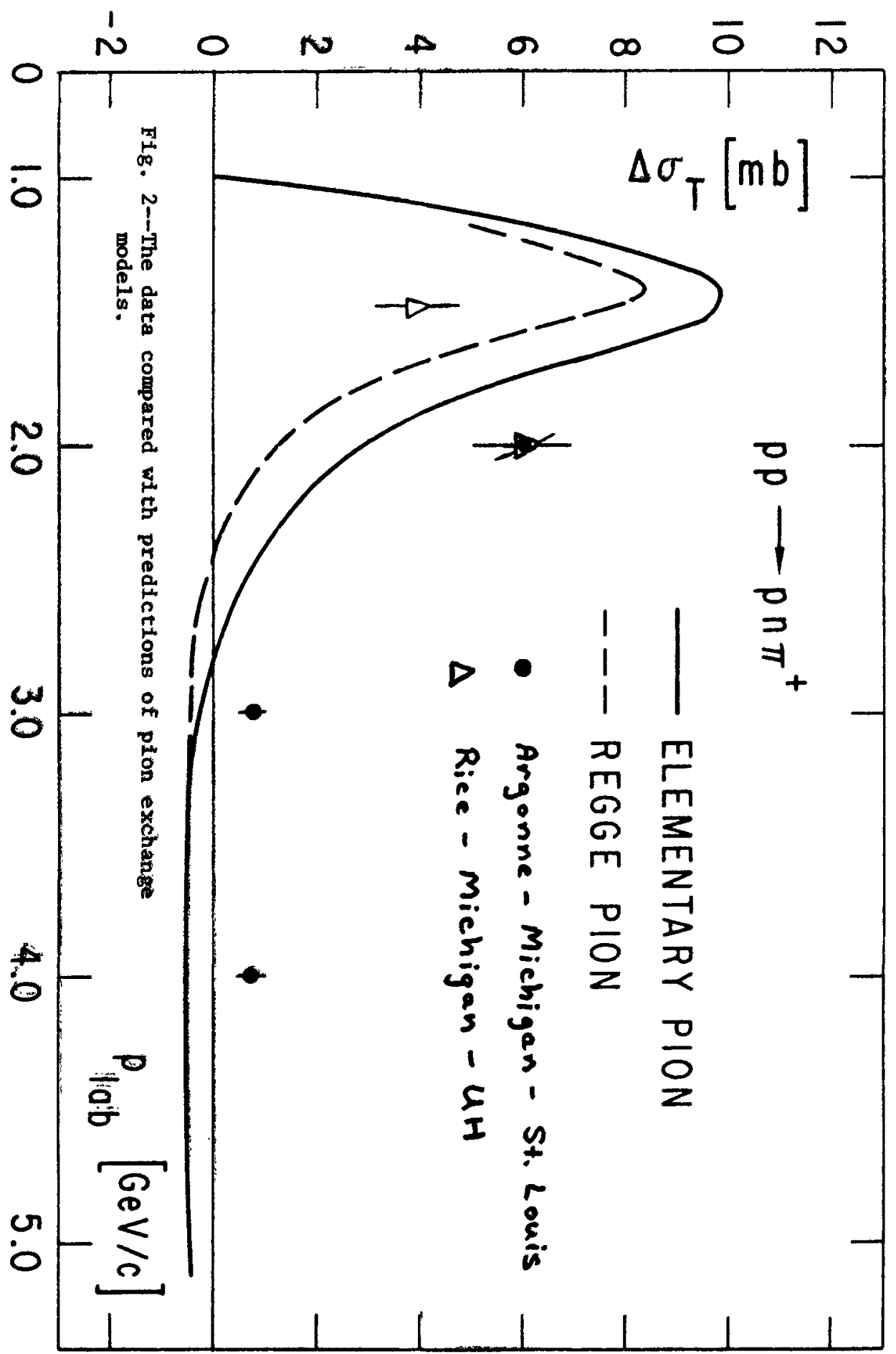




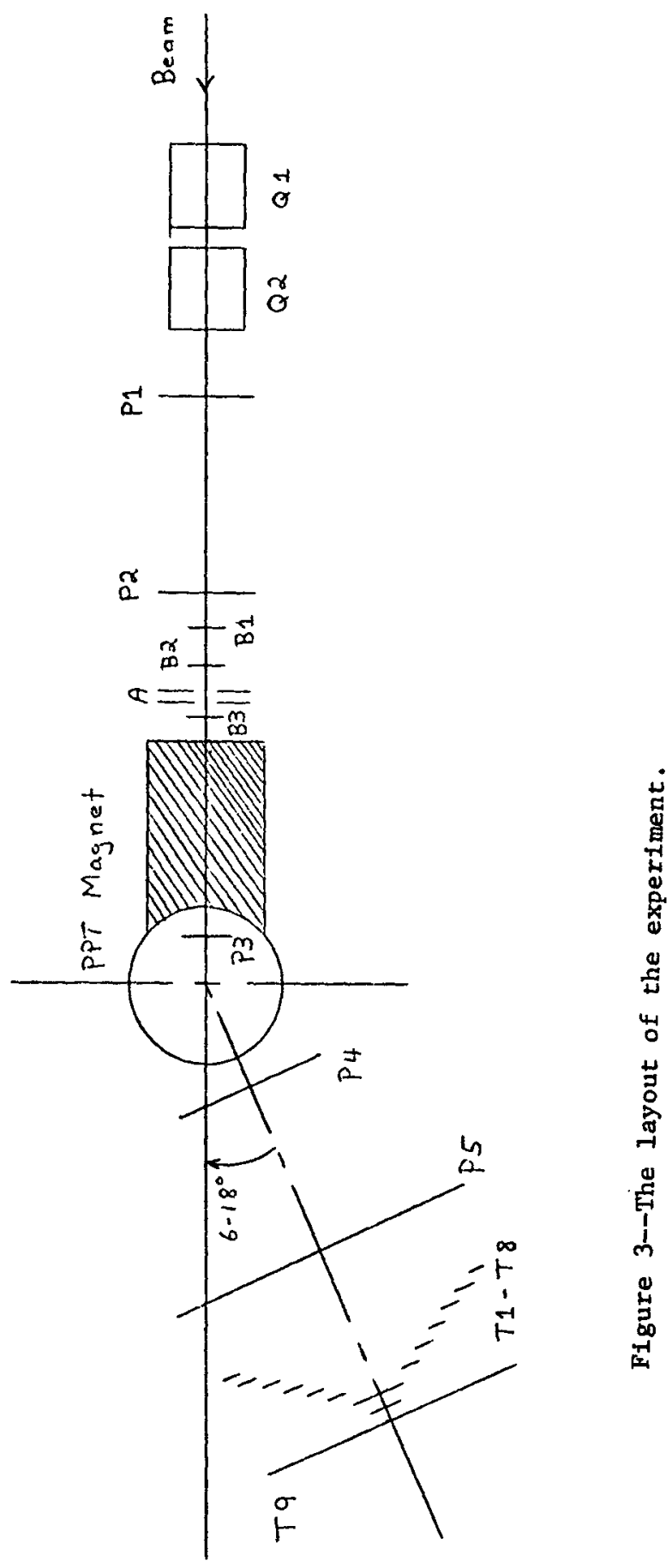

